\title{
SMALL FRACTIONAL PARTS OF QUADRATIC FORMS
}

\author{
by R. C. BAKER and G. HARMAN* \\ (Received 11th February 1981)
}

\section{Introduction}

Let $\|x\|$ denote the distance of $x$ from the nearest integer. In $1948 \mathrm{H}$. Heilbronn proved [5] that for $\varepsilon>0$ and $N>c_{1}(\varepsilon)$ the inequality

$$
\min _{1 \leqq n \leqq N}\left\|\alpha n^{2}\right\|<N^{-(1 / 2)+\varepsilon}
$$

holds for any real $\alpha$. This result has since been generalised in many different directions, and we consider here extensions of the type: For $\varepsilon>0, N>c_{2}(\varepsilon, s)$ and a quadratic form $Q\left(x_{1}, \ldots, x_{s}\right)$ there exist integers $n_{1}, \ldots, n_{s}$ not all zero with $\left|n_{1}\right|, \ldots,\left|n_{s}\right| \leqq N$ and with

$$
\left\|Q\left(n_{1}, \ldots, n_{s}\right)\right\|<N^{-c_{3}(s)+\varepsilon}
$$

Danicic obtained a result of this type [2] with $c_{3}(s)=s /(s+1)$. Cook was able to get (1) with $c_{3}(s)=1$ for an additive form in two variables [1]. More recently, Schinzel, Schlickewei and Schmidt have shown [7] that $c_{3}(s)$ may be taken as the maximum of

$$
2\left(1+\frac{1}{h}+\frac{4}{s-h+1}\right)^{-1}
$$

over odd $h$ in $1 \leqq h \leqq(s+5) / 3$. Taking $h$ asymptotically equal to $s / 3$ gives

$$
c_{3}(s)=2-(18 / s)+O\left(1 / s^{2}\right)
$$

This result improves on Danicic's for $s \geqq 7$ and, as is well known, the "limiting" exponent -2 is best possible. The new idea in [7] is the use of an auxiliary result on quadratic congruences. For a different approach to the limiting result $c_{3}(s) \rightarrow 2$, see [9].

In the present note we refine the method of [7] to prove

Theorem. Let $s \geqq 3$ and let $Q\left(x_{1}, \ldots, x_{s}\right)$ be a real quadratic form. Then there is a constant $c_{4}(s)$ such that for every integer $N \geqq 2$ there are integers $n_{1}, \ldots, n_{s}$ with

$$
0<\max \left(\left|n_{1}\right|, \ldots,\left|n_{s}\right|\right) \leqq N,
$$

*Written while the second author held a University of London postgraduate studentship. 
having

$$
\left\|Q\left(n_{1}, \ldots, n_{s}\right)\right\|<c_{4}(s)(N / \log N)^{-c_{5}(s)}
$$

Here

$$
c_{5}(s)= \begin{cases}2 s /(s+5) & \text { for odd } s \\ 2 s(s-1) /\left(s^{2}+4 s-4\right) & \text { for even } s\end{cases}
$$

Our exponent is the same as Danicic's for $s=3$, apart from the substitution of a power of $\log N$ for $N^{\varepsilon}$. For $s \geqq 4$, our exponent is better than that of [2] or [7], and (4) gives

$$
c_{5}(s)=2-(10 / s)+O\left(1 / s^{2}\right)
$$

The second author has refined the method further for diagonal quadratic forms; for example, one can take $c_{5}(5)=9 / 8$ and $c_{5}(11)=3 / 2$ in this special case.

The key to the improvement on [7] is Lemma 1, below. This is a straightforward extension of the congruence result of [7], but enables us to introduce successive minima explicitly. This is more economical; the procedure is analogous to that of Davenport and Ridout [4].

\section{Quadratic congruences}

Lemma 1. Let $Q(\mathbf{x})=Q\left(x_{1}, \ldots, x_{h}\right)$ be a quadratic form in an odd number $h$ of variables with integer coefficients. Let $m$ be a natural number. Let $K_{1}, \ldots, K_{h}$ be positive reals with

$$
K_{1} \ldots K_{h} \geqq m^{(h+1) / 2}
$$

Then there are integers $x_{1}, \ldots, x_{h}$ not all zero, with

$$
Q\left(x_{1}, \ldots, x_{h}\right) \equiv 0 \quad(\bmod m)
$$

and having

$$
\left|x_{i}\right| \leqq K_{i} \quad(i=1, \ldots, h)
$$

The case $K_{1}=\ldots=K_{h}=m^{(1 / 2)+(1 / 2 h)}$ is Theorem 1 of [7].

Proof. We first observe that the result is trivial if $K_{i} \geqq m$ for some $i$; hence we suppose that

$$
K_{i}<m \quad(i=1, \ldots, h)
$$


Clearly we may assume that $m>1$, and that $m$ is square free. For any $m$ may be written in the form

$$
m=r^{2} a
$$

where $a$ is square free. If $K_{1} \ldots K_{h} \geqq m^{(h+1) / 2}$, then $\left(K_{1} / r\right) \ldots\left(K_{h} / r\right) \geqq a^{(h+1) / 2}$. A solution $\left(y_{1}, \ldots, y_{h}\right)$ of $Q(y) \equiv 0(\bmod a)$, with $\left|y_{i}\right| \leqq K_{i} / r$, yields a solution $x_{i}=r y_{i}$ of $(5)$ satisfying (7).

Let $d=(h-1) / 2$. According to [7], for every prime $p$ dividing $m$ there are integer vectors $\mathbf{r}_{1}^{(p)}, \ldots, \mathbf{r}_{d}^{(p)}$ which are linearly independent modulo $p$, and for which

$$
Q\left(s_{1} \mathbf{r}_{1}^{(p)}+\ldots+s_{d} \mathbf{r}_{d}^{(p)}\right) \equiv 0 \quad(\bmod p)
$$

whenever $s_{1}, \ldots, s_{d}$ are integers. By the Chinese remainder theorem there are integer vectors $\mathbf{r}_{1}, \ldots, \mathbf{r}_{d}$ having

$$
\mathbf{r}_{i} \equiv \mathbf{r}_{i}^{(p)} \quad(\bmod p)
$$

for each prime $p$ dividing $m$. Write $\mathbf{r}_{i}=\left(r_{i 1}, \ldots, r_{i h}\right)$.

By Minkowski's linear forms theorem, and taking account of (5), there are integers $s_{1}, \ldots, s_{d}, z_{1}, \ldots, z_{h}$ not all zero, with

$$
\begin{gathered}
\left|s_{i}\right|<m, \quad(i=1, \ldots, d) \\
\left|\sum_{k=1}^{d} s_{k} r_{k j}+m z_{j}\right| \leqq K_{j} \quad(j=1, \ldots, h) .
\end{gathered}
$$

Put $\mathbf{x}=s_{1} \mathbf{r}_{1}+\ldots+s_{d} \mathbf{r}_{d}+m z$, where $\mathbf{z}=\left(z_{1}, \ldots, z_{h}\right)$. Then clearly (6) holds, and (7) follows from (10). Since $K_{j}<m$ we easily see that $\left(s_{1}, \ldots, s_{h}\right) \neq 0$, say $s_{1} \neq 0$. Since $m$ is square free, there is a prime factor $p$ of $m$ with $s_{1} \neq 0(\bmod p)$. Because $\mathbf{r}_{1}, \ldots, \mathbf{r}_{d}$ are linearly independent $(\bmod p)$, we have $\mathbf{x} \neq 0(\bmod p)$. Thus $\mathbf{x} \neq 0$.

\section{A lemma on exponential sums}

The following lemma was pointed out to us by H. L. Montgomery. Compared with the familiar Lemma 12 of [8], Chapter I, it saves a great deal of work, and a small power of $\log N$, farther on.

Lemma 2. Let $L, M$ be natural numbers and let $\alpha_{1}, \alpha_{2}, \ldots, \alpha_{M}$ be real numbers such that $\left\|\alpha_{n}\right\| \geqq L^{-1}(n=1, \ldots, M)$. Then we have

$$
\sum_{l=1}^{L}\left|\sum_{n=1}^{M} e\left(l \alpha_{n}\right)\right| \geqq M / 6 .
$$


Proof. Let $J=\left(L^{-1}, 1-L^{-1}\right)$ with indicator function $X_{J}(x)$. According to Montgomery [6], p. 559, there is a function $b \in L^{1}(R)$ such that

$$
\begin{gathered}
b(x) \geqq X_{J}(x), \quad \hat{b}(0)=|J|+L^{-1} \\
\hat{b}(t)=0 \quad \text { for } \quad|t| \geqq L .
\end{gathered}
$$

By an easy calculation, the function

$$
B(x)=\sum_{n} b(x+n)
$$

is in $L^{1}(0,1)$ with Fourier series $\sum_{|k| \leqq L} f(k) e(k x)$, hence

$$
B(x)=\sum_{|k| \leqq L} \hat{b}(k) e(k x)
$$

Note that for integral $k \neq 0,(13)$ implies

$$
\begin{aligned}
|\hat{b}(k)| & \leqq \int_{0}^{1}|B(x)-1| d x \leqq \int_{0}^{1}\left\{(B(x)-1)+2\left(1-X_{J}(x)\right)\right\} d x \\
& =\hat{b}(0)+1-2|J|=3 L^{-1}
\end{aligned}
$$

Combining (11), (13) and (14) with the hypothesis $\left\|\alpha_{n}\right\| \geqq L^{-1}$, we obtain

$$
\begin{aligned}
M & \leqq \sum_{n=1}^{M} B\left(\alpha_{n}\right) \leqq M b(0)+\sum_{0<|k| \leqq L}|\hat{b}(k)|\left|\sum_{n=1}^{M} e\left(k \alpha_{n}\right)\right| \\
& \leqq M \hat{b}(0)+6 L^{-1} \sum_{k=1}^{L}\left|\sum_{n=1}^{M} e\left(k \alpha_{n}\right)\right|
\end{aligned}
$$

Since $1-\hat{b}(0)=L^{-1}$, the desired inequality follows.

\section{Proof of the theorem}

The proof will be by contradiction. Suppose that there are no integers $n_{1}, \ldots, n_{s}$ satisfying (2) and (3). Let

$$
S(l)=\sum_{n_{1}=1}^{N} \ldots \sum_{n_{s}=1}^{N} e\left(l Q\left(n_{1}, \ldots, n_{s}\right)\right)
$$

Let

$$
L=\left[2 c_{4}(s)^{-1}(N / \log N)^{c s(s)}\right]
$$


where $c_{4}(s)$ is sufficiently large, then from Lemma 2 with $M=N^{s}$ we have

$$
\sum_{i=1}^{L}|S(l)| \geqq N^{s} / 6
$$

Let $l$ be a natural number, $1 \leqq l \leqq L$, having

$$
|S(l)| \geqq N^{s} / 6 L
$$

We define linear forms $L_{1}, \ldots, L_{s}$ with symmetric coefficient matrix via the identity

$$
Q\left(x_{1}, \ldots, x_{s}\right)=x_{1} L_{1}(\mathbf{x})+\ldots+x_{s} L_{s}(\mathbf{x}) .
$$

Let $M_{1}, \ldots, M_{s}$ be the first $s$ successive minima of the convex body described by

$$
\left.\begin{array}{c}
\left|2 l L_{j}(\mathbf{x})-x_{s+j}\right|<N^{-1} \\
\left|x_{j}\right|<N
\end{array}\right\} \quad(j=1, \ldots, s),
$$

with respect to the integer lattice in $2 s$-dimensional space. It is established in the proof of Lemma 5 of [3] that

$$
|S(l)|^{2} \leqq c_{6}(s)\left(M_{1} \ldots M_{s}\right)^{-1} N^{s}(\log N)^{s}
$$

In view of (15), then,

$$
\left(M_{1} \ldots M_{s}\right)^{-1} \geqq c_{7}(s) L^{-2} N^{s}(\log N)^{-s}
$$

We now consider the cases of odd and even $s$ separately.

Case I. Odd $s$. By the definition of successive minima, we can find $s$ linearly independent integer vectors $\mathbf{r}_{\mu}^{\prime}$ in $2 s$-dimensional space with

$$
\begin{gathered}
\left|2 l L_{j}\left(\mathbf{r}_{\mu}\right)-r_{j+s, \mu}\right|<N^{-1} M_{\mu}, \\
\left|r_{j \mu}\right|<N M_{\mu}
\end{gathered}
$$

for $j=1, \ldots, s, \mu=1, \ldots, s$. Here $\mathbf{r}_{\mu}^{\prime}=\left(r_{1 \mu}, \ldots, t_{2 s, \mu}\right)$ and $\mathbf{r}_{\mu}=\left(r_{1 \mu}, \ldots, r_{s \mu}\right)$.

Let us write

$$
K_{\mu}=c_{7}(s)^{-1 / s} L^{2 / s}(2 l)^{(s+1) / 2 s} M_{\mu}^{-1}(\log N) N^{-1}
$$

then

$$
K_{1} \ldots K_{s} \geqq(2 l)^{(s+1) / 2}
$$

EMS E 
from (16). We also write

$$
\theta_{\mu v}=2 l \sum_{j=1}^{s} r_{j \mu} L_{j}\left(\mathbf{r}_{v}\right) \quad(\mu, v=1, \ldots, s)
$$

so that

$$
\left\|\theta_{\mu v}\right\|<s M_{\mu} M_{v}
$$

from (17) and (18). Let $b_{\mu \nu}$ be integers with

$$
\left\|\theta_{\mu \nu}\right\|=\left|\theta_{\mu \nu}-b_{\mu \nu}\right| \quad(\mu, v=1, \ldots, s) .
$$

By Lemma 1 and (20) there are integers $x_{1}, \ldots, x_{s}$ not all zero, with

$$
\left|x_{\mu}\right| \leqq K_{\mu} \quad(\mu=1, \ldots, s)
$$

and

$$
\sum_{\mu=1}^{s} \sum_{\nu=1}^{s} b_{\mu \nu} x_{\mu} x_{v} \equiv 0 \quad(\bmod 2 l)
$$

Put $n_{i}=\sum_{\mu=1}^{s} r_{i \mu} x_{\mu}$ for $i=1, \ldots, s$. Then

$$
\begin{aligned}
Q\left(n_{1}, \ldots, n_{s}\right) & =\sum_{\mu=1}^{s} \sum_{v=1}^{s}\left(\sum_{i=1}^{s} L_{i}\left(\mathbf{r}_{\mu}\right) r_{i v}\right) x_{\mu} x_{v} \\
& =(2 l)^{-1} \sum_{\mu=1}^{s} \sum_{\nu=1}^{s} \theta_{\mu v} x_{\mu} x_{v} \\
& =(2 l)^{-1} \sum_{\mu=1}^{s} \sum_{v=1}^{s} b_{\mu v} x_{\mu} x_{v}+(2 l)^{-1} \sum_{\mu=1}^{s} \sum_{\nu=1}^{s}\left(\theta_{\mu v}-b_{\mu v}\right) x_{\mu} x_{v}
\end{aligned}
$$

The first sum on the right-hand side of (25) is an integer, in view of (24). Thus

$$
\begin{aligned}
\left\|Q\left(n_{1}, \ldots, n_{s}\right)\right\| & \leqq(2 l)^{-1} \sum_{\mu=1}^{s} \sum_{v=1}^{s}\left\|\theta_{\mu v}\right\|\left|x_{\mu} \| x_{v}\right| \\
& <\frac{s}{2 l} \sum_{\mu=1}^{s} \sum_{v=1}^{s} M_{\mu} M_{v} K_{\mu} K_{v} \\
& =\frac{s^{3}}{2 l}\left(c_{7}(s)\right)^{-2 / s} L^{4 / s}(2 l)^{(s+1) / s}(\log N)^{2} N^{-2}
\end{aligned}
$$


from (21) and (23). For sufficiently large $c_{4}(s)$, we have

$$
\begin{aligned}
\left\|Q\left(n_{1}, \ldots, n_{s}\right)\right\| & <2 s^{3}\left(c_{7}(s)\right)^{-2 / s} L^{5 / s}(N / \log N)^{-2} \\
& <L^{-1}
\end{aligned}
$$

Moreover, we have

$$
\begin{aligned}
\left|n_{i}\right|=\left|\sum_{\mu=1}^{s} r_{i \mu} x_{\mu}\right| & \leqq s M_{\mu} N K_{\mu} \\
& \leqq s c_{7}(s)^{-1 / s} L^{2 / s}(2 l)^{(s+1) / 2 s} \log N \\
& \leqq 2 s c_{7}(s)^{-1 / s} L^{(s+5) / 2 s} \log N<N
\end{aligned}
$$

By hypothesis, then, we must have

$$
\left(n_{1}, \ldots, n_{s}\right)=\mathbf{0}
$$

so that $\sum_{\mu=1}^{s} x_{\mu} \mathbf{r}_{\mu}=0$ and consequently

$$
\sum_{\mu=1}^{s} x_{\mu} L_{j}\left(\mathbf{r}_{\mu}\right)=\mathbf{0} \quad(j=1, \ldots, s) .
$$

Combining (26) with (17) we obtain

$$
\begin{gathered}
\left|\sum_{\mu=1}^{s} x_{\mu} r_{j+s, \mu}\right|<N^{-1} \sum_{\mu=1}^{s} M_{\mu}\left|x_{\mu}\right| \\
\leqq N^{-1} \sum_{\mu=1}^{s} M_{\mu} K_{\mu}<1
\end{gathered}
$$

as we already saw above. Hence

$$
\sum_{\mu=1}^{s} x_{\mu} r_{j \mu}=0
$$

is true not only for $j=1, \ldots, s$ but for $j=s+1, \ldots, 2 s$ also. This contradicts the linear independence of $\mathbf{r}_{1}^{\prime}, \ldots, \mathbf{r}_{\mu}^{\prime}$.

Thus the theorem is proved in Case I.

Case II. Even $s$. From (16) and $M_{1} \leqq \ldots \leqq M_{s}$, we obtain

$$
\left(M_{1} \ldots M_{s-1}\right)^{-1} \geqq c_{7}(s)^{(s-1) / s} L^{-2(s-1) / s}(N / \log N)^{s-1} .
$$


Let $\mathbf{r}_{\mu}^{\prime}, \mathbf{r}_{\mu}, \theta_{\mu \nu}, b_{\mu \nu}$ be as in Case I. By repeating the argument of Case I, with $s-1$ instead of $s$, we obtain integers $x_{1}, \ldots, x_{s-1}$ such that

$$
\sum_{\mu=1}^{s-1} \sum_{v=1}^{s-1} b_{\mu v} x_{\mu} x_{v} \equiv 0 \quad(\bmod 2 l)
$$

and

$$
\left|x_{\mu}\right| \leqq H_{\mu}=c_{8}(s) L^{2 / s}(2 l)^{s / 2(s-1)} M_{\mu}^{-1}(\log N) N^{-1}
$$

After all,

$$
H_{1} \ldots H_{s-1} \geqq(2 l)^{((s-1) / 2)+1 / 2}
$$

provided that $c_{8}(s)$ is sufficiently large. Let

$$
\left(n_{1}, \ldots, n_{s}\right)=\sum_{\mu=1}^{s-1} x_{\mu} \mathbf{r}_{\mu}
$$

Continuing as before, we obtain for $\left\|Q\left(n_{1}, \ldots, n_{s}\right)\right\|$ the upper bound

$$
\begin{aligned}
\frac{s^{3}}{2 l}\left(\max _{1 \leqq \mu \leqq s-1} H_{\mu} M_{\mu}\right)^{2} & \leqq c_{9}(s) L^{(4 / s)+(1 /(s-1))}(\log N)^{2} N^{-2} \\
& <L^{-1}
\end{aligned}
$$

and

$$
\begin{aligned}
\max \left(\left|n_{1}\right|, \ldots,\left|n_{s}\right|\right) & \leqq s \max _{1 \leqq \mu \leqq s-1} H_{\mu} M_{\mu} N \\
& \leqq c_{10}(s) L^{(2 / s)+(s / 2(s-1))} \log N<N,
\end{aligned}
$$

for a suitable choice of $c_{4}(s)$. The argument used in Case I can be repeated to obtain

$$
\sum_{\mu=1}^{s-1} x_{\mu} \mathbf{r}_{\mu}^{\prime}=\mathbf{0}
$$

which is a contradiction. This proves the theorem in Case II.

\section{REFERENCES}

1. R. J. Соoк, On the fractional parts of an additive form, Proc. Camb. Philos. Soc. 72 (1972), 209-212.

2. I. Danicic, An extension of a theorem of Heilbronn, Mathematika 5 (1958), 30-37.

3. H. Davenport, Indefinite quadratic forms in many variables (II), Proc. London Math. Soc. (3) 8 (1958), 109-126. 
4. H. Davenport and D. Ridout, Indefinite quadratic forms, Proc. London Math. Soc. (3) 9 (1959), 544-555.

5. H. Heilbronn, On the distribution of the sequence $\theta n^{2}(\bmod 1)$, Quart. J. Math. 19 (1948), 249-256.

6. H. L. Montgomery, The analytic principle of the large sieve, Bull. Amer. Math. Soc. 84 (1978), 547-567.

7. A. Schinzel, H.-P. Schlickewei and W. M. Schmidt, Small solutions of quadratic congruences and small fractional parts of quadratic forms, Acta Arithmetica 37 (1980), 241-248.

8. I. M. Vinogradov, The Method of Trigonometric Sums in the Theory of Numbers (WileyInterscience, New York, 1954).

9. R. J. Cook, Small fractional parts of quadratic forms in many variables, Mathematika 27 (1980), 25-29.

\section{Royal Holloway College}

EGHAM

SURREY 